\title{
Synthesis and Characterization of Novel Thieno-Fused Bicyclic Compounds through New Enaminone Containing Thieno[2,3-b]pyridine Scaffold
}

\author{
Yahia Nasser Mabkhot, ${ }^{1}$ Salah S. Aladdi, ${ }^{1}$ S. S. Al-Showiman, ${ }^{1}$ \\ A. M. A. Al-Majid, ${ }^{1}$ Assem Barakat, ${ }^{1,2}$ Hazem A. Ghabbour, ${ }^{3,4}$ and Mohamed R. Shaaban ${ }^{5,6}$ \\ ${ }^{1}$ Department of Chemistry, College of Science, King Saud University, P.O. Box 2455, Riyadh 11451, Saudi Arabia \\ ${ }^{2}$ Department of Chemistry, Faculty of Science, Alexandria University, P.O. Box 426, Ibrahimia, Alexandria 21321, Egypt \\ ${ }^{3}$ Department of Pharmaceutical Chemistry, College of Pharmacy, King Saud University, P.O. Box 2457, Riyadh 11451, Saudi Arabia \\ ${ }^{4}$ Department of Medicinal Chemistry, Faculty of Pharmacy, Mansoura University, Mansoura 35516, Egypt \\ ${ }^{5}$ Department of Chemistry, Faculty of Science, Cairo University, Giza 12613, Egypt \\ ${ }^{6}$ Department of Chemistry, Faculty of Applied Science, Umm Al-Qura University, Makkah Al-Mukarramah 21955, Saudi Arabia
}

Correspondence should be addressed to Yahia Nasser Mabkhot; yahia@ksu.edu.sa

Received 6 October 2015; Accepted 2 November 2015

Academic Editor: Josefina Pons

Copyright (C) 2015 Yahia Nasser Mabkhot et al. This is an open access article distributed under the Creative Commons Attribution License, which permits unrestricted use, distribution, and reproduction in any medium, provided the original work is properly cited.

\begin{abstract}
New substituted thieno-fused bicyclic compounds named ( $1 H$-pyrazol-3-yl)thieno[2,3-b]pyridin-4(7H)-one derivatives (4a,b), 2 $([1,2,4]$ triazolo[1,5-a]pyrimidin-7-yl)-3-methyl-7-phenylthieno[2,3-b]pyridin-4(7H)-one (5), phenylthieno[2,3- $b]$ pyridin-4(7H)one derivatives (6a-c, 7a-c, and 8), and 3-(3-methyl-4-oxo-7-phenyl-4,7-dihydrothieno[2,3-b]pyridin-2-yl)-3-oxopropyl 4chlorobenzoate (9) were synthesized by reacting the new enaminone (3) with different reagents. The chemical structure of the new molecules was determined by means of different spectroscopic methods such as NMR, IR, MS spectrometry, and by CHN analyses. The molecular structure of the 1,1'-(3-methyl-5-(phenylamino)thiophene-2,4-diyl)diethanone (2) was successfully solved by X-ray single crystal.
\end{abstract}

\section{Introduction}

E naminones are a precursor for the synthesis of heterocycles which have been devoted in the last decade. These synthons were reacted with several nucleophilic and electrophilic reagents to construct new heterocyclic systems of pharmaceutical targets such as pyrazolo[3,4-d]pyrimidin-4one, pyrazolo[1,5-a]pyrimidine derivatives [1], pyrido[2,3- $d]$ $[1,2,4]$ triazolino[4,3-a]pyrimidin-4-one, pyridino[2,3- $d]$ pyrimidin-4-one, and pyrido[2,3-d] [1,2,4]triazolino[4,5- $a]$ pyrimidin-5-one [2]. Thieno-fused bicyclic compounds incorporate pyrimidine, pyrazoles, benzo[4,5]imidazo[1,2- $a]$ pyrimidine and $[1,2,4]$ triazolo[1,5-a]pyrimidine rings [3], isoquinoline [4], and natural products and their analogues $[5,6]$.
Additionally, the thieno[2,3-b]pyridine core structure and its substituents are scaffold possessing potential biological activities including estrogenic, antihypertensive anti-inflammatory, antiviral, anticancer, and antimicrobial activities [7-15]; indeed, many substituted thieno[2,3$b$ ]pyridine building blocks were synthesized and recognized for the selectivity of these bioisosteres of the antibacterial quinolones such as ofloxacin, ciprofloxacin, and norfloxacin $[16,17]$. In continuation of our interest in the chemistry of thieno-fused bicyclic compounds [18-21] to develop new library of pharmaceutical targets we started from new enaminone 3 as precursor for synthesis of new thienofused bicyclic compounds containing thieno $[2,3-b]$ pyridine core. 


\section{Experimental Section}

2.1. General. All the chemical products were purchased from various suppliers, including Sigma-Aldrich and Fluka, and were used without further purification, unless otherwise stated. All melting points were measured on a Gallenkamp melting point apparatus in open glass capillaries and are uncorrected. IR spectra were recorded as $\mathrm{KBr}$ pellets on a Nicolet 6700 FT-IR spectrophotometer. The NMR spectra were recorded on a Varian Mercury Jeol-400 NMR spectrometer. ${ }^{1} \mathrm{HNMR}(400 \mathrm{MHz})$ and ${ }^{13} \mathrm{C}-\mathrm{NMR}(100 \mathrm{MHz})$ were run in deuterated dimethyl sulphoxide (DMSO- $\left.d_{6}\right)$. Chemical shifts $(\delta)$ are referred to in ppm while J-coupling constants were represented in Hz. Mass spectra were recorded on a Jeol of JMS-600 H. Elemental analysis was carried out on Elmer 2400 Elemental Analyzer, CHN mode.

1,1'-(3-Methyl-5-(phenylamino)thiophene-2,4-diyl)diethanone (2). In a round bottom flask $(100 \mathrm{~mL})$ acetylacetone $(10 \mathrm{~mL}$, $100 \mathrm{mmol}$ ) was dissolved in DMF $(45 \mathrm{~mL})$; then the reaction mixture was kept at RT for 5 mins; subsequently, phenyl isocyanate $(13.5 \mathrm{~mL}, 100 \mathrm{mmol})$ was added and stirred for further 30 mins. the reaction mixture was then cooled by using ice bath, and chloroacetone $(9.25 \mathrm{~mL}, 100 \mathrm{mmol})$ was added and stirred for 15 mins. The reaction mixture was allowed to warm to rt and continued stirring for 30 mins. The precipitated product was filtered off and washed by $\mathrm{H}_{2} \mathrm{O}$ and dried. The crude product was purified by crystallization from glacial acetic acid to afford the pure compound in yellow crystals.

Yield: 95\%; m.p. $115^{\circ} \mathrm{C}$; IR $\left(\nu_{\max }\right): 1492,1602-1646(\mathrm{C}=\mathrm{O})$, $3415(\mathrm{NH}) ;{ }^{1} \mathrm{H}-\mathrm{NMR}\left(\mathrm{DMSO}-d_{6}\right): \delta 2.85\left(\mathrm{~s}, 3 \mathrm{H}, \mathrm{CH}_{3}\right), 3.38$ $\left(\mathrm{s}, 6 \mathrm{H}, 2 \mathrm{CH}_{3}\right), 5.85(\mathrm{~s}, 1 \mathrm{H}, \mathrm{NH}), 7.63-7.84\left(\mathrm{~m}, 5 \mathrm{H}, \mathrm{C}_{6} \mathrm{H}_{5}\right) ;{ }^{13} \mathrm{C}-$ NMR: $\delta, 16.08\left(\mathrm{CH}_{3}\right), 40,46\left(\mathrm{COCH}_{3}\right), 120.19,122.46,130.55$, (Ar-C), $137.04\left(\mathrm{CH}_{3} \mathrm{COC}\right), 140.95$ (Ar-C-N), $142.34\left(\mathrm{CH}_{3}-\mathrm{C}\right)$; MS m/z (\%): 274 [M+, 100]; Anal. calcd. for $\mathrm{C}_{15} \mathrm{H}_{15} \mathrm{NO}_{2} \mathrm{~S}: \mathrm{C}$, 65.91; H, 5.53; N, 5.12; S, 11.73; Found: C, 65.92; H, 5.55; N, $5.10 ; \mathrm{S}, 11.70$.

2-(3-(Dimethylamino)acryloyl)-3-methyl-7-phenylthieno[2,3b]pyridin-4(7H)-one (3). A mixture of 1,1'-(3-methyl-5(phenylamino)thiophene-2,4-diyl)diethanone 2 (1.37 g, $0.5 \mathrm{mmol})$ and DMF-DMA $(1.19 \mathrm{~mL}, 0.01 \mathrm{~mol})$ was refluxed in $m$-xylene ( $15 \mathrm{~mL}$ ) for $3 \mathrm{~h}$. After cooling, the resulting solid product was collected by filtration to give the desired product 3 used for the next step without any further purification.

Yellow crystal; Yield: 63\%; m.p. $235^{\circ} \mathrm{C}$; IR $\left(\nu_{\max }\right): 1593$ $(\mathrm{C}=\mathrm{C}), 1613-1640(\mathrm{C}=\mathrm{O}) . ;{ }^{1} \mathrm{H}-\mathrm{NMR}\left(\mathrm{DMSO}-d_{6}\right): \delta, 2.85(\mathrm{~s}$, $\left.3 \mathrm{H}, \mathrm{CH}_{3}\right), 3.16\left(\mathrm{~s}, 6 \mathrm{H}, \mathrm{N}\left(\mathrm{CH}_{3}\right)_{2}\right), 5.36\left(\mathrm{~d}, 1 \mathrm{H}, \mathrm{CH}_{\mathrm{a}^{\prime}}\right) 5.39(\mathrm{~d}$, $\left.1 \mathrm{H}, \mathrm{CH}_{\mathrm{b}^{\prime}}\right), 6.14\left(\mathrm{~d}, 1 \mathrm{H}, \mathrm{CH}_{\mathrm{a}}\right) 6.16\left(\mathrm{~d}, 1 \mathrm{H}, \mathrm{CH}_{\mathrm{b}}\right), 7.63-7.68(\mathrm{~m}$, $\left.5 \mathrm{H}, \mathrm{C}_{6} \mathrm{H}_{5}\right) ;{ }^{13} \mathrm{C}-\mathrm{NMR}: \delta, 16.12\left(-\mathrm{CH}_{3}\right), 45.15\left(-\mathrm{N}=\left(\mathrm{CH}_{3}\right)_{2}\right)$, $94.03(-\mathrm{CH}=\mathrm{CH}-\mathrm{N}=), 153.15\left(-\mathrm{CH}_{\mathrm{b}^{\prime}}\right), 126.35,128.58,130.55$ (Ph), $142.35\left(-\mathrm{C}-\mathrm{CH}_{3}\right), 115.14\left(-\mathrm{CH}_{\mathrm{a}}\right), 154.45\left(-\mathrm{CH}_{\mathrm{b}}\right), 154.54$ (=CNS), 176.49, 180.51 (C=O); MS m/z (\%): 325 [M+, 30\%]; Anal. calcd. for $\mathrm{C}_{18} \mathrm{H}_{17} \mathrm{~N}_{2} \mathrm{O}_{2} \mathrm{~S}$ : C, 67.43; H, 5.36; N, 8.28; S, 9.47; Found: C, 67.45; H, 5.40; N, 8.31; S, 9.50.

2.2. General Procedure for the Preparation of Compounds $4 \boldsymbol{a}, \boldsymbol{b}$. Compounds $\mathbf{4 a}, \mathbf{b}$ were prepared using the following general procedure.
Compound 3 ( $0.131 \mathrm{~g}, 0.5 \mathrm{mmol})$ was added to hydrazine hydrate $(2 \mathrm{~mL}, 63 \mathrm{mmol})$ or phenylhydrazine $(1 \mathrm{~mL}, 10 \mathrm{mmol})$ (to make compounds $\mathbf{4 a}, \mathbf{b}$, resp.). The mixture of compound 3 and the appropriate hydrazine was refluxed until compound 3 is completely dissolved, followed by addition of the mixed solvent EtOH/DMF. The reaction mixture was then refluxed for $6-7 \mathrm{~h}$ and the product was filtered after cooling. The desired product was obtained in a pure form by recrystallization from $\mathrm{MeOH}$. Using the general procedure, the following compounds were prepared.

3-Methyl-7-phenyl-2-(1H-pyrazol-3-yl)thieno[2,3-b]pyridin4(7H)-one (4a). Red crystal; Yield: 67\%; m.p. $285^{\circ} \mathrm{C}$; IR $\left(\nu_{\max }\right): 1616(\mathrm{C}=\mathrm{N}), 1637(\mathrm{C}=\mathrm{O}), 3477(\mathrm{NH}) ; \mathrm{MS} \mathrm{m} / z(\%): 322$ [M+, 30\%]; Anal. calcd. for $\mathrm{C}_{18} \mathrm{H}_{16} \mathrm{~N}_{3} \mathrm{OS}$ : C, 66.43; H, 4.26; N, 13.67; S, 10.43; Found: C, 66.41; H, 4.25; N, 13.66; S, 10.41.

3-Methyl-7-phenyl-2-(1-phenyl-1H-pyrazol-3-yl)thieno[2,3b]pyridin-4(7H)-one (4b). Green crystal; Yield: 68\%; m.p. $275^{\circ} \mathrm{C}$; IR $\left(\nu_{\max }\right): 1602(\mathrm{C}=\mathrm{N}), 1640(\mathrm{C}=\mathrm{O}) ;{ }^{1} \mathrm{H}-\mathrm{NMR}$ $\left(\mathrm{DMSO}-d_{6}\right): \delta, 2.29\left(\mathrm{~s}, 3 \mathrm{H}, \mathrm{CH}_{3}\right), 6.14-6.16\left(\mathrm{~d}, 1 \mathrm{H}, \mathrm{CH}_{\mathrm{a}}\right)$, 6.72-6.74 (d, $\left.1 \mathrm{H}, \mathrm{CH}_{\mathrm{a}^{\prime}}\right), 7.63-7.65\left(\mathrm{~d}, 1 \mathrm{H}, \mathrm{CH}_{\mathrm{b}^{\prime}}\right), 7.67-7.78$ $\left(\mathrm{d}, 1 \mathrm{H}, \mathrm{CH}_{\mathrm{b}}\right), 7.61-7.69\left(\mathrm{~m}, 5 \mathrm{H}, \mathrm{C}_{6} \mathrm{H}_{5}\right) ;{ }^{13} \mathrm{C}-\mathrm{NMR}: \delta, 15.09$ $\left(\mathrm{CH}_{3}\right), 113.23\left(\mathrm{CH}_{\mathrm{a}}\right), 119.70,126.31,129.49$ (Ar-C), 130.49 (-C-CH ${ }_{3}$ ), 130.93 (N-CHCS), 131.04 (-C-S), 140.39 (C-CO), 142.42 (Ar-C-N), $145.95\left(\mathrm{CH}_{\mathrm{b}}\right), 175.74(\mathbf{C}=\mathrm{O}) ; \mathrm{MS} \mathrm{m} / z(\%)$ : 307 [M+, 30\%]; Anal. calcd. for $\mathrm{C}_{17} \mathrm{H}_{13} \mathrm{~N}_{3} \mathrm{OS}$ : C, 72.04; $\mathrm{H}$, 4.47; N, 10.96; S, 8.36; Found: C, 72.04; H, 4.49; N, 11.0; S, 8.37.

2-([1,2,4]Triazolo[1,5-a]pyrimidin-7-yl)-3-methyl-7-phenylthieno[2,3-b]pyridin-4(7H)-one (5). It is a mixture of $3(0.131 \mathrm{~g}$, $0.5 \mathrm{mmol}$ ) and 3-amine- $1 \mathrm{H}-1,2,4$-triazole $(0.042 \mathrm{~g}, 0.5 \mathrm{mmol})$ in dioxane. Catalytic amount of TEA (triethyl amine) and $\mathrm{ZnCl}_{2}$ was added into the reaction mixture. The reaction mixture was then refluxed for $6 \mathrm{~h}$ and the product was filtered after cooling.

Yellow crystal; Yield: $72 \%$; m.p. $>300^{\circ} \mathrm{C}$; IR $\left(\nu_{\max }\right): 1618$ $(\mathrm{C}=\mathrm{N}), 1634(\mathrm{C}=\mathrm{O}) ; \mathrm{MS} m / z(\%): 359$ [M+, 30\%]; Anal. calcd. for $\mathrm{C}_{19} \mathrm{H}_{13} \mathrm{~N}_{5} \mathrm{OS}$ : C, 63.50; H, 3.65; N, 19.49; S, 8.92; Found: C, 63.51; H, 3.65; N, 19.50; S, 8.93.

2.3. General Procedure for the Preparation of Compounds $\mathbf{6} \boldsymbol{a}-\boldsymbol{c}$. Compounds $\mathbf{6 a}-\mathbf{c}$ were prepared using the following general procedure.

Compound $3(0.131 \mathrm{~g}, 0.5 \mathrm{mmol})$ was added to ethane1,2-diamine (0.03 g, $0.5 \mathrm{mmol})$, propane-1,3-diamine $(0.04 \mathrm{~g}$, $0.5 \mathrm{mmol}$ ), or hexane-1,2-diamine $(0.06 \mathrm{~g}, 0.5 \mathrm{mmol})$ (to make compounds $\mathbf{6 a}-\mathbf{c}$, resp. $)$ in $\mathrm{AcOH}(0.1 \mathrm{mmol})$ as solvent and $\mathrm{NH}_{4} \mathrm{OAc}(0.1 \mathrm{mmol})$ as catalyst. The reaction mixture was then refluxed for $3 \mathrm{~h}$ and the product was filtered after cooling. The desired product was obtained in a pure form by recrystallization from EtOH. Using the general procedure, the following compounds were prepared.

2-(3-((2-Aminoethyl)amino)acryloyl)-3-methyl-7-phenylthieno[2,3-b]pyridin-4(7H)-one (6a). Yellow powder; Yield: 62\%; m.p. $142^{\circ} \mathrm{C}$; IR $\left(\nu_{\max }\right): 1593(\mathrm{C}=\mathrm{C}), 1616-1637(2 \mathrm{C}=\mathrm{O})$, $2931\left(\mathrm{CH}_{2}\right), 3238\left(\mathrm{NH}_{2}\right) 3415(\mathrm{NH}) ;{ }^{1} \mathrm{H}-\mathrm{NMR}\left(\mathrm{DMSO}-d_{6}\right)$ : 
$\delta 2.63\left(\mathrm{~s}, 3 \mathrm{H}, \mathrm{CH}_{3}\right), 2.93\left(\mathrm{q}, 2 \mathrm{H}, \mathrm{CH}_{2} \mathrm{NH}\right), 3.48(\mathrm{~m}, 2 \mathrm{H}$, $\left.\mathrm{CH}_{2} \mathrm{NH}_{2}\right), 6.16\left(\mathrm{~s}, 2 \mathrm{H}, \mathrm{NH}_{2}\right), 5.24-5.40\left(\mathrm{~d}, 1 \mathrm{H}, \mathrm{CH}_{\mathrm{a}^{\prime}}\right)$, 5.83-5.91 (d, $\left.1 \mathrm{H}, \mathrm{CH}_{\mathrm{a}}\right), 7.02-7.00\left(\mathrm{~m}, 1 \mathrm{H}, \mathrm{CH}_{\mathrm{b}^{\prime}}\right), 7.08-7.20$ $\left(\mathrm{d}, 1 \mathrm{H}, \mathrm{CH}_{\mathrm{b}}\right.$ ), 7.64-7.67 (m, 5H, $\left.\mathrm{C}_{6} \mathrm{H}_{5}\right) ;{ }^{13} \mathrm{C}-\mathrm{NMR}: \delta 115.20$ $\left(\mathrm{CH}_{2} \mathrm{NH}_{2}\right)$, $115.52\left(\mathrm{CH}_{2}-\mathrm{CH}=\mathrm{CH}\right), 129.31,130.91,130.94$, 143.28, (Ar-C), $131.04\left(\mathrm{CH}_{\mathrm{a}^{\prime}}\right), 137.45$ (=CCO), 141.62 (SCCO), $142.31\left(-\mathrm{C}-\mathrm{CH}_{3}\right), 143.28(\mathrm{Ar}-\mathrm{C}-\mathrm{N}), 153.19\left(\mathrm{CH}_{\mathrm{b}}\right)$, $155.15\left(\mathrm{CH}_{\mathrm{b}^{\prime}}\right), 155.74\left(\mathrm{CH}_{\mathrm{a}}\right), 176.48(\mathrm{C}=\mathrm{O}) ; \mathrm{MS} \mathrm{m} / z(\%): 353$ [M+, 30\%]; Anal. calcd. for $\mathrm{C}_{18} \mathrm{H}_{14} \mathrm{~N}_{2} \mathrm{O}_{2} \mathrm{~S}: \mathrm{C}, 64.57 ; \mathrm{H}, 5.42$; N, 11.89; S, 9.07; Found: C, 64.56; H, 5.40; N, 11.91; S, 9.10.

2-(3-((3-Aminopropyl)amino)acryloyl)-3-methyl-7-phenylthieno[2,3-b]pyridin-4(7H)-one $(\mathbf{6} \boldsymbol{b})$. Yellow powder; Yield: 64\%; m.p. $145^{\circ} \mathrm{C}$; IR $\left(\nu_{\max }\right): 1593(\mathrm{C}=\mathrm{C}), 1616-1637(2 \mathrm{C}=\mathrm{O})$, $2931\left(\mathrm{CH}_{2}\right), 3238\left(\mathrm{NH}_{2}\right) 3415(\mathrm{NH}) ;{ }^{1} \mathrm{H}-\mathrm{NMR}\left(\mathrm{DMSO}-d_{6}\right): \delta$ $2.74\left(\mathrm{~s}, 3 \mathrm{H}, \mathrm{CH}_{3}\right), 3.16\left(\mathrm{t}, 2 \mathrm{H}, \mathrm{NH}_{2}\right), 1.30-2.95\left(\mathrm{~m}, 6 \mathrm{H}, \mathrm{CH}_{2}\right)$, 6.08-6.20 (m, 1H, $\left.\mathrm{CH}_{\mathrm{a}^{\prime}}\right), 6.17-6.20\left(\mathrm{~d}, 1 \mathrm{H}, \mathrm{CH}_{\mathrm{a}}\right), 7.64-7.43(\mathrm{~d}$, $\left.1 \mathrm{H}, \mathrm{CH}_{\mathrm{b}^{\prime}}\right), 9.90-7.80\left(\mathrm{~d}, 1 \mathrm{H}, \mathrm{CH}_{\mathrm{b}}\right), 7.62-7.72\left(\mathrm{~m}, 5 \mathrm{H}, \mathrm{C}_{6} \mathrm{H}_{5}\right)$; ${ }^{13}$ C-NMR: $\delta, 13.93\left(\mathrm{CH}_{3}\right), 28.50\left(-\mathrm{CH}_{2}\right), 33.00\left(-\mathrm{CH}_{2} \mathrm{NH}_{2}\right)$, $69.92\left(-\mathrm{CH}_{2}-\mathrm{NH}\right) 114.60\left(\mathrm{CH}_{\mathrm{a}}\right), 126.36,129.18,130.13(\mathrm{Ar}-\mathrm{C})$, $131.06\left(\mathrm{CH}_{\mathrm{a}^{\prime}}\right), 136.05$ (=CCO), $139.77\left(-\mathrm{C}-\mathrm{CH}_{3}\right), 142.57$ $\left(\mathrm{CH}_{\mathrm{b}}\right), 151.40\left(\mathrm{CH}_{\mathrm{b}^{\prime}}\right), 175.74(\mathrm{C}=\mathrm{O}) ; \mathrm{MS} \mathrm{m} / z(\%): 367[\mathrm{M}+$, $30 \%$; Anal. calcd. for $\mathrm{C}_{28} \mathrm{H}_{26} \mathrm{~N}_{2} \mathrm{O}_{2} \mathrm{~S}_{2}$ : C, 65.37; $\mathrm{H}, 5.76$; N, 11.44; S, 8.72; Found: C, 65.35; H, 5.77; N, 11.45; S, 8.74.

2-(3-((4-Aminobutyl)amino)acryloyl)-3-methyl-7-phenylthieno[2,3-b]pyridin-4(7H)-one (6c). Yellow powder; Yield: 67\%; m.p. $282^{\circ} \mathrm{C}$; IR $\left(\nu_{\max }\right): 1593(\mathrm{C}=\mathrm{C}), 1616-1637(2 \mathrm{C}=\mathrm{O})$, $2931\left(\mathrm{CH}_{2}\right), 3238\left(\mathrm{NH}_{2}\right) 3415(\mathrm{NH}) ;{ }^{1} \mathrm{H}-\mathrm{NMR}\left(\mathrm{DMSO}-d_{6}\right): \delta$ $2.74\left(\mathrm{~s}, 3 \mathrm{H}, \mathrm{CH}_{3}\right), 1.15-2.87\left(\mathrm{~m}, 6 \mathrm{H}, \mathrm{CH}_{2}\right), 3.80\left(\mathrm{t}, 2 \mathrm{H}, \mathrm{NH}_{2}\right)$, $6.18\left(\mathrm{q}, 1 \mathrm{H}, \mathrm{CH}_{\mathrm{a}}\right), 6.19\left(\mathrm{~d}, 1 \mathrm{H}, \mathrm{CH}_{\mathrm{a}^{\prime}}\right), 6.20\left(\mathrm{~d}, 1 \mathrm{H}, \mathrm{CH}_{\mathrm{b}}\right), 6.20$ $\left(\mathrm{d}, 1 \mathrm{H}, \mathrm{CH}_{\mathrm{b}^{\prime}}\right), 7.58-7.67\left(\mathrm{~m}, 5 \mathrm{H}, \mathrm{C}_{6} \mathrm{H}_{5}\right) ;{ }^{13} \mathrm{C}-\mathrm{NMR}: \delta 13.93$ $\left(\mathrm{CH}_{3}\right), 28.50\left(-\mathrm{CH}_{2}\right), 33.00\left(-\mathrm{CH}_{2} \mathrm{NH}_{2}\right), 69.92\left(-\mathrm{CH}_{2}-\mathrm{NH}\right)$ $114.60\left(\mathrm{CH}_{\mathrm{a}}\right), 126.36,129.18,130.13(\mathrm{Ar}-\mathrm{C}), 131.06\left(\mathrm{CH}_{\mathrm{a}^{\prime}}\right)$, 136.05 (=CCO), 139.77(-C-CH $\left.{ }_{3}\right), 142.57 \quad\left(\mathrm{CH}_{\mathrm{b}}\right), \quad 151.40$ $\left(\mathrm{CH}_{\mathrm{b}^{\prime}}\right), 175.74(\mathrm{C}=\mathrm{O})$; MS m/z (\%): 409 [M+, 30\%]; Anal. calcd. for $\mathrm{C}_{23} \mathrm{H}_{24} \mathrm{~N}_{2} \mathrm{O}_{2} \mathrm{~S}$ : C, 66.12; H, 6.08; N, 11.01; S, 8.40; Found: C, 66.12; H, 6.11; N, 11.04; S, 8.41.

2.4. General Procedure for the Preparation of Compounds $7 \boldsymbol{a}-\boldsymbol{c}$. Compounds $7 \mathbf{a}-\mathbf{c}$ were prepared using the following general procedure.

Compound 3 (0.131 g, $0.5 \mathrm{mmol})$ was added to 4 chloroaniline $(0.06 \mathrm{~g}, 0.5 \mathrm{mmol}), 4$-methoxyaniline $(0.06 \mathrm{~g}$, $0.5 \mathrm{mmol}$ ), or $p$-toluidine $(0.05 \mathrm{~g}, 0.5 \mathrm{mmol}$ ) (to make compounds $\mathbf{6 a}-\mathbf{c}$, resp.) in dioxane as solvent and TEA (triethyl amine) $(0.1 \mathrm{mmol})$ as catalyst. The reaction mixture was then refluxed for $4 \mathrm{~h}$ and the product was filtered after cooling. The desired product was obtained in a pure form by recrystallization from $\mathrm{EtOH}$.

2-(3-((4Chlorohphenyl)amino)acryloyl)-3-methyl-7-phenylthieno[2,3-b]pyridin-4(7H)-one (7a). Yellow powder; Yield: 58\%; m.p. $>300^{\circ} \mathrm{C}$; IR $\left(v_{\max }\right): 1578(\mathrm{C}=\mathrm{C}), 1636(\mathrm{C}=\mathrm{O}), 3415$ $(\mathrm{NH}) ;{ }^{1} \mathrm{H}-\mathrm{NMR}\left(\mathrm{DMSO}-\mathrm{d}_{6}\right): \delta 2.49\left(\mathrm{~s}, 3 \mathrm{H}, \mathrm{CH}_{3}\right), 5.21(\mathrm{~d}$, $1 \mathrm{H}, \mathrm{NH}), 6.51-6.53\left(\mathrm{~d}, 1 \mathrm{H}, \mathrm{CH}_{\mathrm{a}^{\prime}}\right), 6.55-6.57\left(\mathrm{~d}, 1 \mathrm{H}, \mathrm{CH}_{\mathrm{a}}\right)$, $6.97\left(\mathrm{~d}, 1 \mathrm{H}, \mathrm{CH}_{\mathrm{a}}\right), 6.97-6.99\left(\mathrm{t}, 1 \mathrm{H}, \mathrm{CH}_{\mathrm{b}^{\prime}}\right), 7.01-7.03(\mathrm{t}, 1 \mathrm{H}$, $\left.\mathrm{CH}_{\mathrm{b}}\right), 7.78-7.82\left(\mathrm{~m}, 4 \mathrm{H}, \mathrm{C}_{6} \mathrm{H}_{4}\right) ;{ }^{13} \mathrm{C}-\mathrm{NMR}: \delta 115.70,119.21$, 129.03 (Ar-C), 148.25 (Ar-C-N); MS m/z (\%): $420.5[\mathrm{M}+$,
30\%]; Anal. calcd. for $\mathrm{C}_{23} \mathrm{H}_{15} \mathrm{ClN}_{2} \mathrm{O}_{2}$ S: C, 65.63; $\mathrm{H}, 4.07 ; \mathrm{Cl}$, 8.42; N, 6.66; S, 7.62.; Found: C, 65.64; H, 4.09; Cl, 8.40; N, $6.67 ; \mathrm{S}, 7.63$.

2-(3-((4-Methoxyphenyl)amino)acryloyl)-3-methyl-7-phenylthieno[2,3-b]pyridin-4(7H)-one (7b). Yellow powder; Yield: $47 \%$; m.p. $>300^{\circ} \mathrm{C}$; IR $\left(\nu_{\max }\right): 1578(\mathrm{C}=\mathrm{C}), 1636(\mathrm{C}=\mathrm{O}), 3415$ $(\mathrm{NH}) ;{ }^{1} \mathrm{H}-\mathrm{NMR}\left(\mathrm{DMSO}-d_{6}\right): \delta, 2.50\left(\mathrm{~s}, 3 \mathrm{H}, \mathrm{CH}_{3}\right), 3.61(\mathrm{~s}, 3 \mathrm{H}$, $\left.\mathrm{OCH}_{3}\right), 4.62(\mathrm{~d}, 1 \mathrm{H}, \mathrm{NH}), 6.50-6.52\left(\mathrm{~d}, 1 \mathrm{H}, \mathrm{CH}_{\mathrm{a}^{\prime}}\right), 6.62-6.65$ $\left(\mathrm{d}, 1 \mathrm{H}, \mathrm{CH}_{\mathrm{a}}\right), 6.95-6.98\left(\mathrm{t}, 1 \mathrm{H}, \mathrm{CH}_{\mathrm{b}^{\prime}}\right), 7.69-7.82\left(\mathrm{t}, 1 \mathrm{H}, \mathrm{CH}_{\mathrm{b}}\right)$, 7.78-7.82 (m, 9H, $\left.\mathrm{C}_{6} \mathrm{H}_{4}\right) ;{ }^{13} \mathrm{C}-\mathrm{NMR}: \delta, 56.00\left(\mathrm{OCH}_{3}\right), 115.02$, 115.57 (Ar-C), 142.76 (SCCO), 151.24 (Ar-C-N). MS m/z (\%): 417 [M+, 30\%]; Anal. calcd. for $\mathrm{C}_{24} \mathrm{H}_{18} \mathrm{~N}_{2} \mathrm{O}_{3} \mathrm{~S}: \mathrm{C}, 69.21 ; \mathrm{H}$, 4.84; N, 6.73; S, 7.70; Found: C, 69.20; H, 4.84; N, 6.74; S, 7.70 .

3-Methyl-7-phenyl-2-(3-(p-tolylamino)acryloyl)thieno[2,3b]pyridin-4(7H)-one (7c). Gray powder; Yield: 59\%; m.p. $250^{\circ} \mathrm{C}$; IR $\left(\nu_{\max }\right): 1578(\mathrm{C}=\mathrm{C}), 1636(\mathrm{C}=\mathrm{O}), 3415(\mathrm{NH})$; ${ }^{1} \mathrm{H}-\mathrm{NMR}\left(\mathrm{DMSO}-d_{6}\right): \delta 1.28-3.37\left(\mathrm{~s}, 6 \mathrm{H}, \mathrm{CH}_{3}\right), 4.29(\mathrm{~d}, 1 \mathrm{H}$, $\mathrm{NH}), 4.26-4.28\left(\mathrm{~d}, 1 \mathrm{H}, \mathrm{CH}_{\mathrm{a}^{\prime}}\right), 4.29-4.31\left(\mathrm{~d}, 1 \mathrm{H}, \mathrm{CH}_{\mathrm{a}}\right), 6.90$ $\left(\mathrm{t}, 1 \mathrm{H}, \mathrm{CH}_{\mathrm{b}^{\prime}}\right), 7.00-7.05\left(\mathrm{t}, 1 \mathrm{H}, \mathrm{CH}_{\mathrm{b}}\right), 7.78-7.826 .98-7.12(\mathrm{~m}$, $\left.4 \mathrm{H}, \mathrm{C}_{6} \mathrm{H}_{4}\right) .{ }^{13} \mathrm{C}-\mathrm{NMR}: \delta 14.66\left(\mathrm{CH}_{3}\right), 114.06,115.11,122.96$, $(\operatorname{Ar}-\mathrm{C}), 114.06\left(\mathrm{Ch}_{\mathrm{a}}\right), 129.77\left(\mathrm{Ch}_{\mathrm{b}}\right), 143.23$ (SCCO), 143.23 (Ar-C-N), 159.92 (S-C-N); MS m/z (\%): 400 [M+, 30\%]; Anal. calcd. for $\mathrm{C}_{24} \mathrm{H}_{18} \mathrm{~N}_{2} \mathrm{O}_{2} \mathrm{~S}$ : C, 71.98; H, 5.03; N, 6.99; S, 8.01.; Found: C, 72.00; H, 5.01; N, 7.00; S, 8.00.

2-(Benzo[4,5]imidazo[1,2-a]pyrimidin-4-yl)-3-methyl-7-phenylthino[2,3-b]pyridin-4(7H)-one (8). It is a mixture of $3(0.131 \mathrm{~g}, 0.5 \mathrm{mmol})$ and 2-aminobenzimidazole $(0.07 \mathrm{~g}$, $0.5 \mathrm{mmol}$ ) in EtOH as solvent and TEA (triethyl amine)/ $\mathrm{ZnCl}_{2}$ as catalyst. The reaction mixture was then refluxed for $8 \mathrm{~h}$ and the product was filtered after cooling. The desired product was obtained in a pure form.

Red crystal, Yield: $62 \%$; m.p. $210^{\circ} \mathrm{C}$; IR $\left(\nu_{\max }\right): 1588(\mathrm{C}=\mathrm{N})$, $1628(\mathrm{C}=\mathrm{O}) ;{ }^{1} \mathrm{H}-\mathrm{NMR}\left(\mathrm{DMSO}-d_{6}\right): \delta 2.75\left(\mathrm{~s}, 3 \mathrm{H}, \mathrm{CH}_{3}\right), 5.48-$ $5.51\left(\mathrm{~d}, 1 \mathrm{H}, \mathrm{CH}_{\mathrm{a}^{\prime}}\right), 6.24-6.26\left(\mathrm{~d}, 1 \mathrm{H}, \mathrm{CH}_{\mathrm{a}}\right), 7.10-7.27(\mathrm{t}, 1 \mathrm{H}$, $\left.\mathrm{CH}_{\mathrm{b}^{\prime}}\right), 7.87-8.02\left(\mathrm{t}, 1 \mathrm{H}, \mathrm{CH}_{\mathrm{b}}\right), 7.71-7.79\left(\mathrm{~m}, 9 \mathrm{H}, \mathrm{C}_{6} \mathrm{H}_{5}\right) .{ }^{13} \mathrm{C}-$ NMR: $\delta, 15.47\left(\mathrm{CH}_{3}\right), 114.83\left(\mathrm{CH}_{\mathrm{a}}\right), 121.17,125.80,126.16$, 130.19, 130.58, 140.62, 142.50 (Ar-C), $125.80\left(\mathrm{CH}_{\mathrm{a}^{\prime}}\right), 126.16$ $\left(\mathrm{CH}_{\mathrm{b}}\right), 130.68\left(\mathrm{~S}-\mathrm{C}=\mathrm{CH}_{3}\right), 162.50(\mathrm{~N}-\mathrm{C}=\mathrm{N}($ pyrazole $)), 162.21$ $\left(\mathrm{CH}_{\mathrm{b}^{\prime}}\right), 162.50\left(\mathrm{~N}-\mathrm{C}=\mathrm{CCH}_{3}\right), 176.50(\mathrm{~S}-\mathrm{C}-\mathrm{N}), 189.64(\mathrm{C}=\mathrm{O})$. MS $m / z$ (\%): 422 [M+, 30\%]; Anal. calcd. for $\mathrm{C}_{25} \mathrm{H}_{19} \mathrm{~N}_{4} \mathrm{OS}$ : C, 65.88; H, 4.07; N, 16.17; S, 9.25; Found: C, 65.85; H, 4.06; $\mathrm{N}, 16.18 ; \mathrm{S}, 9.25$.

3-(3-Methyl-4-oxo-7-phenyl-4,7-dihydrothieno[2,3-b]pyridin-2-yl)-3-oxoprop-1-en-1-yl 4-chlorobenzoate (9). It is a mixture of $3(0.131 \mathrm{~g}, 0.5 \mathrm{mmol})$ and 4-chlorobenzoic acid $(0.08 \mathrm{~g}, 0.5 \mathrm{mmol})$ in dioxane as solvent and TEA (triethyl amine) $(0.1 \mathrm{mmol})$ as catalyst. The reaction mixture was then refluxed for $4 \mathrm{~h}$ and the product was filtered after cooling. The desired product was obtained in a pure form by recrystallization from $\mathrm{EtOH}$.

Yellow powder, Yield: 67\%; m.p. $265^{\circ} \mathrm{C}$; IR $\left(\nu_{\max }\right): 1594$ $(\mathrm{C}=\mathrm{C}), 1610-1630(3 \mathrm{C}=\mathrm{O}) .{ }^{1} \mathrm{H}-\mathrm{NMR}\left(\mathrm{DMSO}-d_{6}\right): \delta 3.12(\mathrm{~s}$, $\left.3 \mathrm{H}, \mathrm{CH}_{3}\right), 5.36-5.39\left(\mathrm{~d}, 1 \mathrm{H}, \mathrm{CH}_{\mathrm{a}^{\prime}}\right), 6.15-6.17\left(\mathrm{~d}, 1 \mathrm{H}, \mathrm{CH}_{\mathrm{a}}\right)$, 7.66-7.68 (t, $\left.1 \mathrm{H}, \mathrm{CH}_{\mathrm{b}^{\prime}}\right), 7.70-7.80\left(\mathrm{t}, 1 \mathrm{H}, \mathrm{CH}_{\mathrm{b}}\right), 7.66-7.68(\mathrm{~m}$, 


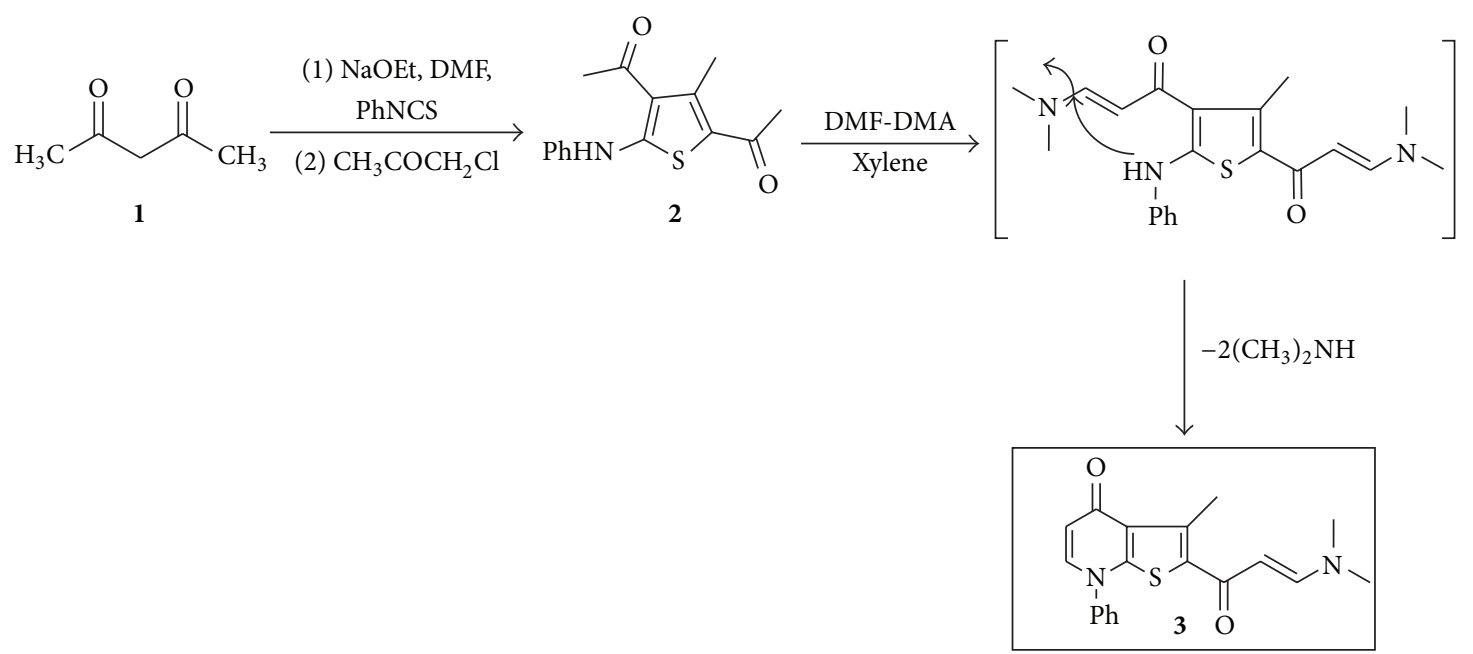

SCHEME 1: Synthesis of compound 3.

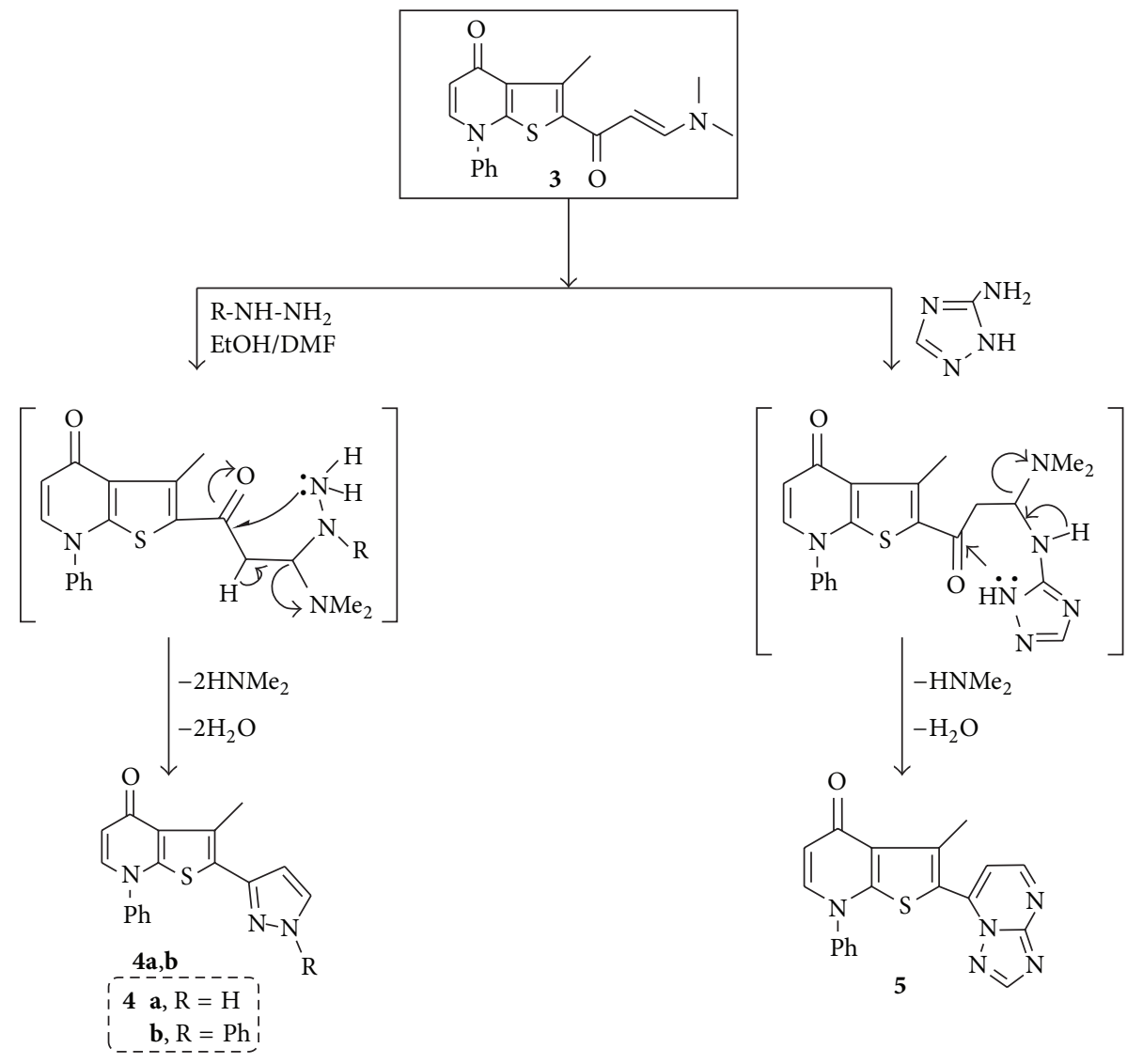

SCHeme 2: Synthesis of compounds $\mathbf{4 a}, \mathbf{b}$ and $\mathbf{5}$.

5H, $\left.\mathrm{C}_{6} \mathrm{H}_{5}\right) ;{ }^{13} \mathrm{C}-\mathrm{NMR}: \delta, 16.11\left(\mathrm{CH}_{3}\right), 115.13\left(\mathrm{CH}_{\mathrm{a}}\right), 126.33$ $\left(\mathrm{CH}_{, \mathrm{a}^{\prime}}\right), 128.58,130.54,130.95,132.60,137.24$ (Ar-C), 141.03 (S-CCO), $142.34\left(-\mathbf{C}-\mathrm{CH}_{3}\right), 153.16\left(\mathrm{CH}_{\mathrm{b}^{\prime}}\right), 154.52(\mathrm{~S}-\mathrm{C}-\mathrm{N})$, 176.51 (COO), $180.51(\mathrm{C}=\mathrm{O})$; MS $\mathrm{m} / z(\%): 444$ [M+, 30\%]; Anal. calcd. for $\mathrm{C}_{24} \mathrm{H}_{14} \mathrm{ClNO}_{4} \mathrm{~S}$ : C, 64.36; H, 3.15; Cl, 7.91; N, 3.13; S, 7.16; Found: C, 64.37; H, 3.14; Cl, 7.91; N, 3.15; S, 7.18 .

\section{Results and Discussion}

Novel thieno-fused bicyclic compounds $\mathbf{4 a}, \mathbf{b}, \mathbf{5}, \mathbf{6 a}-\mathbf{c}, 7 \mathbf{a}-$ c, 8, and 9 were synthesized as shown in Schemes 2 and 3 starting from the new enaminone 3 (Scheme 1). The new synthesized molecules 1-9 were characterized by $\mathrm{CHN}$ analyses, NMR, and MS spectral data. 
<smiles>Cc1c(C(=O)/C=C/N[Al])sc2c1c(=O)ccn2-c1ccccc1</smiles><smiles>Cc1c(C(=O)/C=C/NC(N)N)sc2c1c(=O)ccn2-c1ccccc1</smiles>

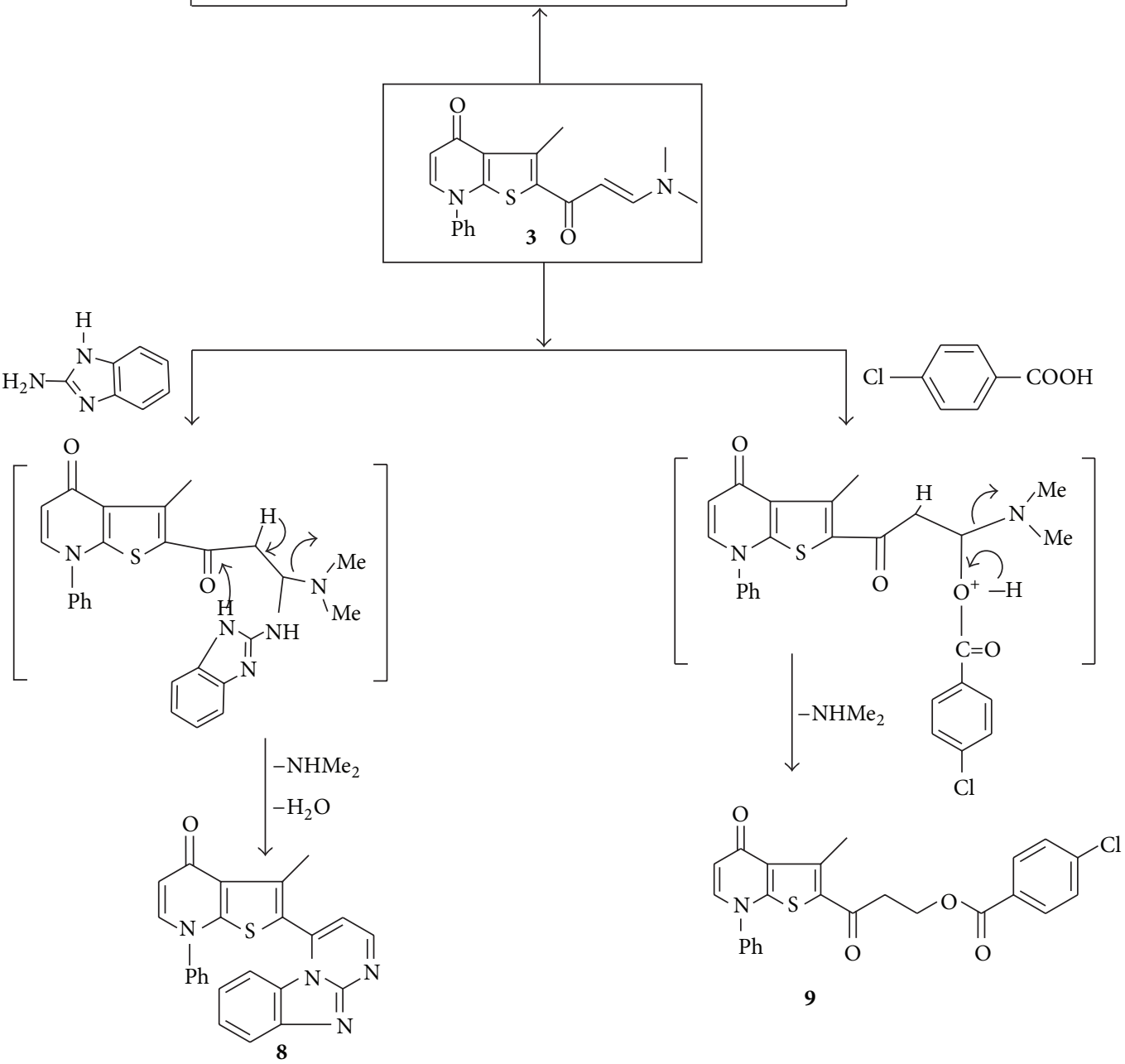

SCHEME 3: Synthesis of compounds 6-9.

The starting intermediate $\mathbf{2}$ needed for this research proposal was synthesized by reported method by Mabkhot et al. [22]. The new enaminone 3 was synthesized via the reaction of acetyl thiophene 2 with DMF-DMA in xylene as solvent as shown in Scheme 1. The final product $\mathbf{3}$ was presumably formed as a result of intramolecular conjugate addition of the nucleophilic nitrogen onto the further end of the double bond ( $\beta$-carbon) of the intermediate bisenaminone with elimination of a dimethylamine molecule resulting in formation of Michael product $\mathbf{3}$ as depicted in Scheme 1 .
The IR spectrum of the new enaminone $\mathbf{3}$ has shown an absorption band at $1613-1640 \mathrm{~cm}^{-1}$ ascribed to the carbonyl group $v(\mathrm{C}=\mathrm{O})$ and an abruption band at $1593 \mathrm{~cm}^{-1}$ ascribed to the alkene group $v(\mathrm{C}=\mathrm{C})$.

${ }^{1} \mathrm{H}-\mathrm{NMR}$ spectrum of the new enaminone 3 exhibited a singlet at $\delta 2.85$ and $3.15 \mathrm{ppm}$, due to the methyl of the $\mathrm{N}\left(\mathrm{CH}_{3}\right)_{2}$ groups. In addition, two doublets for the $\mathrm{CH}=\mathrm{CH}$ protons at $5.36(\mathrm{~J}=11.6 \mathrm{~Hz})$ and $5.39(\mathrm{~J}=11.6 \mathrm{~Hz})$ were observed. On the other hand, two doublets for the $\mathrm{CH}=\mathrm{CH}$ protons at $6.14(\mathrm{~J}=8.0 \mathrm{~Hz})$ and $6.16(\mathrm{~J}=8.0 \mathrm{~Hz})$ due to pyridinone ring in addition to a multiplet at $\delta 7.63-7.68$ 
TABLE 1

\begin{tabular}{|c|c|}
\hline \multicolumn{2}{|c|}{ Crystal data } \\
\hline $\mathrm{C}_{15} \mathrm{H}_{15} \mathrm{NO}_{2} \mathrm{~S}$ & $\gamma=79.233(1)^{\circ}$ \\
\hline$M_{r}=273.35$ & $V=2635.84(16) \AA^{3}$ \\
\hline Triclinic, $P-1$ & $Z=8$ \\
\hline$a=10.3859(3) \AA$ & Mo $K \alpha$ radiation \\
\hline$b=13.8790(6) \AA$ & $\mu=0.24 \mathrm{~mm}^{-1}$ \\
\hline$c=18.8006(6) \AA$ & $T=100 \mathrm{~K}$ \\
\hline$\alpha=86.920(1)^{\circ}$ & $0.59 \times 0.23 \times 0.20 \mathrm{~mm}$ \\
\hline$\beta=82.081(1)^{\circ}$ & \\
\hline \multicolumn{2}{|c|}{ Data collection } \\
\hline $\begin{array}{l}\text { Bruker APEX-II D8 venture } \\
\text { diffractometer }\end{array}$ & $R_{\mathrm{int}}=0.069$ \\
\hline 66528 measured reflections & $\theta_{\max }=30.0^{\circ}$ \\
\hline 15378 independent reflections & Standard reflections: 0 \\
\hline \multicolumn{2}{|c|}{9027 reflections with $I>2 \sigma(I)$} \\
\hline \multicolumn{2}{|c|}{ Refinement } \\
\hline$R\left[F^{2}>2 \sigma\left(F^{2}\right)\right]=0.069$ & 0 restraints \\
\hline$w R\left(F^{2}\right)=0.203$ & $\begin{array}{l}\mathrm{H} \text { atoms treated by a } \\
\text { mixture of independent } \\
\text { and constrained } \\
\text { refinement }\end{array}$ \\
\hline$S=1.09$ & $\Delta \rho_{\max }=1.45 \mathrm{e}^{-3}$ \\
\hline 15378 reflections & $\Delta \rho_{\min }=-0.43 \mathrm{e} \AA^{-3}$ \\
\hline 713 parameters & \\
\hline
\end{tabular}

belong to phenyl ring protons. ${ }^{13} \mathrm{C}$-NMR spectrum agrees well with the suggested structure and showed the following signals: $\delta 16.12\left(-\mathrm{CH}_{3}\right), 45.15\left(-\mathrm{N}=\left(\mathrm{CH}_{3}\right)_{2}\right), 94.03(-\mathrm{CH}=\mathrm{CH}-$ $\mathrm{N}=), 153.15\left(-\mathrm{CH}_{\mathrm{b}^{\prime}}\right), 126.35,128.58,130.55(\mathrm{Ph}), 142.35(-$ C- $\left.\mathrm{CH}_{3}\right), 115.14\left(-\mathrm{CH}_{\mathrm{a}}\right), 154.45\left(-\mathrm{CH}_{\mathrm{b}}\right), 154.54(=\mathrm{CNS})$, and $176.49,180.51(2 \mathrm{C}=\mathrm{O})$. The GC-MS showed the molecular ion $[\mathrm{M}]^{+}$at $\mathrm{m} / \mathrm{z} 325$ corresponding to the molecular formula $\mathrm{C}_{18} \mathrm{H}_{17} \mathrm{~N}_{2} \mathrm{O}_{2} \mathrm{~S}$.

3.1. X-Ray Crystal Structure of 2. The compound, $\mathrm{C}_{15} \mathrm{H}_{15} \mathrm{NO}_{2} \mathrm{~S}$, CCDC: 141975 , crystallizes in triclinic, P1 system with the following unit cell $a=10.3859$ (3) $\AA, b=$ 13.8790 (6) $\AA, c=18.8006$ (6) $\AA, \alpha=86.920$ (1),$\beta=82.081$ $(1)^{\circ}$, and $\gamma=79.233(1)^{\circ}$. The unit cell contains four molecules. The molecule consists of tetrasubstituted thiophene ring, in the C3 and C5 two acetyl groups with different orientation. On the other hand, C4 has methyl group and C6 has amine derivative (Figure 1). In the crystal packing the molecules linked via five nonclassical hydrogen bonds were shown in Figure 2 and Table 3 . The selected data collection, handling, and bond lengths and angles for the crystal were shown in Tables 1 and 2, respectively $[23,24]$.

Having the new enaminone 3 available in our hands encourage us to use it for the synthesis of the thieno-fused bicyclic compounds. Compounds $\mathbf{4 a}, \mathbf{b}$ were synthesized by refluxing a mixture of enaminone 3 and $N$-nucleophile, for example, hydrazine analogous and subsequently heated under reflux in EtOH/DMF as solvent. (Scheme 2). The IR
TABLE 2: Bond lengths and bond angles of compound $2\left(\AA,^{\circ}\right)$.

\begin{tabular}{|c|c|c|c|}
\hline S1A-C3A & $1.749(3)$ & $\mathrm{O} 1 \mathrm{C}-\mathrm{C} 2 \mathrm{C}$ & $1.226(3)$ \\
\hline S1A-C6A & $1.724(2)$ & $\mathrm{O} 2 \mathrm{C}-\mathrm{C} 8 \mathrm{C}$ & $1.237(3)$ \\
\hline S1B-C6B & $1.720(2)$ & $\mathrm{O} 1 \mathrm{D}-\mathrm{C} 2 \mathrm{D}$ & $1.228(3)$ \\
\hline $\mathrm{S} 1 \mathrm{~B}-\mathrm{C} 3 \mathrm{~B}$ & $1.751(3)$ & $\mathrm{O} 2 \mathrm{D}-\mathrm{C} 8 \mathrm{D}$ & $1.234(3)$ \\
\hline S1C-C6C & $1.722(2)$ & $\mathrm{N} 1 \mathrm{~A}-\mathrm{C} 10 \mathrm{~A}$ & $1.422(3)$ \\
\hline $\mathrm{S} 1 \mathrm{C}-\mathrm{C} 3 \mathrm{C}$ & $1.749(3)$ & N1A-C6A & $1.349(3)$ \\
\hline S1D-C6D & $1.721(2)$ & $\mathrm{N} 1 \mathrm{~B}-\mathrm{C} 10 \mathrm{~B}$ & $1.419(3)$ \\
\hline S1D-C3D & $1.752(3)$ & N1B-C6B & $1.346(3)$ \\
\hline $\mathrm{O} 1 \mathrm{~A}-\mathrm{C} 2 \mathrm{~A}$ & $1.229(3)$ & $\mathrm{N} 1 \mathrm{C}-\mathrm{C} 6 \mathrm{C}$ & $1.351(3)$ \\
\hline $\mathrm{O} 2 \mathrm{~A}-\mathrm{C} 8 \mathrm{~A}$ & $1.235(3)$ & $\mathrm{N} 1 \mathrm{C}-\mathrm{C} 10 \mathrm{C}$ & $1.420(3)$ \\
\hline $\mathrm{O} 1 \mathrm{~B}-\mathrm{C} 2 \mathrm{~B}$ & $1.228(3)$ & N1D-C10D & $1.420(3)$ \\
\hline $\mathrm{O} 2 \mathrm{~B}-\mathrm{C} 8 \mathrm{~B}$ & $1.235(3)$ & N1D-C6D & $1.348(3)$ \\
\hline C3A-S1A-C6A & $91.88(11)$ & $\mathrm{O} 2 \mathrm{~B}-\mathrm{C} 8 \mathrm{~B}-\mathrm{C} 5 \mathrm{~B}$ & $120.7(2)$ \\
\hline C3B-S1B-C6B & $91.85(12)$ & $\mathrm{O} 2 \mathrm{~B}-\mathrm{C} 8 \mathrm{~B}-\mathrm{C} 9 \mathrm{~B}$ & $118.5(2)$ \\
\hline $\mathrm{C} 3 \mathrm{C}-\mathrm{S} 1 \mathrm{C}-\mathrm{C} 6 \mathrm{C}$ & $91.81(12)$ & $\mathrm{N} 1 \mathrm{~B}-\mathrm{C} 10 \mathrm{~B}-\mathrm{C} 15 \mathrm{~B}$ & $118.1(3)$ \\
\hline C3D-S1D-C6D & $91.82(12)$ & N1B-C10B-C11B & $121.2(3)$ \\
\hline C6A-N1A-C10A & $123.7(2)$ & $\mathrm{O} 1 \mathrm{C}-\mathrm{C} 2 \mathrm{C}-\mathrm{C} 3 \mathrm{C}$ & $122.8(2)$ \\
\hline C6B-N1B-C10B & $125.1(2)$ & $\mathrm{O} 1 \mathrm{C}-\mathrm{C} 2 \mathrm{C}-\mathrm{C} 1 \mathrm{C}$ & $120.1(2)$ \\
\hline C6C-N1C-C10C & $124.6(2)$ & $\mathrm{S} 1 \mathrm{C}-\mathrm{C} 3 \mathrm{C}-\mathrm{C} 4 \mathrm{C}$ & 111.79 (18) \\
\hline C6D-N1D-C10D & $123.6(2)$ & $\mathrm{S} 1 \mathrm{C}-\mathrm{C} 3 \mathrm{C}-\mathrm{C} 2 \mathrm{C}$ & $117.51(17)$ \\
\hline $\mathrm{O} 1 \mathrm{~A}-\mathrm{C} 2 \mathrm{~A}-\mathrm{C} 1 \mathrm{~A}$ & $120.6(2)$ & S1C-C6C-C5C & $112.18(18)$ \\
\hline $\mathrm{O} 1 \mathrm{~A}-\mathrm{C} 2 \mathrm{~A}-\mathrm{C} 3 \mathrm{~A}$ & $122.4(2)$ & S1C-C6C-N1C & $121.02(18)$ \\
\hline S1A-C3A-C2A & $117.20(18)$ & $\mathrm{N} 1 \mathrm{C}-\mathrm{C} 6 \mathrm{C}-\mathrm{C} 5 \mathrm{C}$ & $126.8(2)$ \\
\hline $\mathrm{S} 1 \mathrm{~A}-\mathrm{C} 3 \mathrm{~A}-\mathrm{C} 4 \mathrm{~A}$ & $111.67(18)$ & $\mathrm{O} 2 \mathrm{C}-\mathrm{C} 8 \mathrm{C}-\mathrm{C} 9 \mathrm{C}$ & $118.3(3)$ \\
\hline S1A-C6A-C5A & $112.22(18)$ & $\mathrm{O} 2 \mathrm{C}-\mathrm{C} 8 \mathrm{C}-\mathrm{C} 5 \mathrm{C}$ & $120.7(2)$ \\
\hline N1A-C6A-C5A & $127.6(2)$ & N1C-C10C-C15C & $118.0(2)$ \\
\hline S1A-C6A-N1A & $120.15(18)$ & N1C-C10C-C11C & $121.0(2)$ \\
\hline $\mathrm{O} 2 \mathrm{~A}-\mathrm{C} 8 \mathrm{~A}-\mathrm{C} 9 \mathrm{~A}$ & $117.7(2)$ & O1D-C2D-C1D & $120.6(2)$ \\
\hline $\mathrm{O} 2 \mathrm{~A}-\mathrm{C} 8 \mathrm{~A}-\mathrm{C} 5 \mathrm{~A}$ & $120.2(2)$ & O1D-C2D-C3D & $122.5(2)$ \\
\hline N1A-C10A-C11A & $120.3(2)$ & $\mathrm{S} 1 \mathrm{D}-\mathrm{C} 3 \mathrm{D}-\mathrm{C} 2 \mathrm{D}$ & $117.06(18)$ \\
\hline $\mathrm{N} 1 \mathrm{~A}-\mathrm{C} 10 \mathrm{~A}-\mathrm{C} 15 \mathrm{~A}$ & $120.6(2)$ & S1D-C3D-C4D & $111.96(18)$ \\
\hline $\mathrm{O} 1 \mathrm{~B}-\mathrm{C} 2 \mathrm{~B}-\mathrm{C} 3 \mathrm{~B}$ & $122.6(2)$ & N1D-C6D-C5D & $127.3(2)$ \\
\hline $\mathrm{O} 1 \mathrm{~B}-\mathrm{C} 2 \mathrm{~B}-\mathrm{C} 1 \mathrm{~B}$ & $120.4(2)$ & S1D-C6D-C5D & $112.21(18)$ \\
\hline S1B-C3B-C4B & $111.87(18)$ & S1D-C6D-N1D & $120.51(18)$ \\
\hline $\mathrm{S} 1 \mathrm{~B}-\mathrm{C} 3 \mathrm{~B}-\mathrm{C} 2 \mathrm{~B}$ & $117.38(17)$ & $\mathrm{O} 2 \mathrm{D}-\mathrm{C} 8 \mathrm{D}-\mathrm{C} 5 \mathrm{D}$ & $120.0(2)$ \\
\hline N1B-C6B-C5B & $126.5(2)$ & O2D-C8D-C9D & $117.7(3)$ \\
\hline S1B-C6B-C5B & $112.20(18)$ & N1D-C10D-C11D & $120.9(2)$ \\
\hline S1B-C6B-N1B & $121.26(19)$ & N1D-C10D-C15D & $120.4(2)$ \\
\hline
\end{tabular}

spectrum of the synthesized molecules exhibited absorption bands at 3416,1640 , and $1602 \mathrm{~cm}^{-1}$ due to $v(\mathrm{~N}-\mathrm{H}), v(\mathrm{C}=\mathrm{O})$, and $\nu(\mathrm{C}=\mathrm{N})$, respectively.

Next, enaminone 3 reacted with 3-amino- $1 H-1,2,4-$ triazole in dioxane in the presence of TEA (triethyl amine) and $\mathrm{ZnCl}_{2}$ as catalyst to afford the final product 2([1,2,4] triazolo[1,5-a]pyrimidin-7-yl)-3-methyl-7-phenylthieno[2,3-b]pyridin-4(7H)-one (5). The suggested chemical structure agreed with experimental spectral data.

In addition, compounds $6 \mathbf{a}-\mathbf{c}, 7 \mathbf{a}-\mathbf{c}$, and $\mathbf{8}$ were synthesized by refluxing a mixture of $\mathbf{3}$ and aliphatic diamines, aromatic amines, and 2-aminobenzimidazole, respectively (Scheme 3). 
TABLE 3: Hydrogen-bond geometry for compound $2\left(\AA,^{\circ}\right)$.

\begin{tabular}{|c|c|c|c|c|}
\hline $\mathrm{D}-\mathrm{H} \cdots \mathrm{A}$ & $\mathrm{D}-\mathrm{H}$ & $\mathrm{H} \cdots \mathrm{A}$ & $\mathrm{D} \cdots \mathrm{A}$ & $\mathrm{D}-\mathrm{H} \cdots \mathrm{A}$ \\
\hline N1D-H1ND $\cdots$ O2C & $0.81(3)$ & $2.37(3)$ & $3.069(3)$ & $145(3)$ \\
\hline N1A-H1NA $\cdots$ O2B & $0.78(3)$ & $2.37(3)$ & $3.057(3)$ & $149(3)$ \\
\hline $\mathrm{N} 1 \mathrm{C}-\mathrm{H} 1 \mathrm{NC} \cdots \mathrm{O} 2 \mathrm{C}$ & $0.85(4)$ & $1.98(3)$ & $2.651(3)$ & $135(3)$ \\
\hline N1C-H1NC $\cdots$ O2D & $0.85(4)$ & $2.31(4)$ & $2.968(3)$ & $135(3)$ \\
\hline N1B-H1NB $\cdots$ O2A & $0.80(3)$ & $2.36(3)$ & $2.987(3)$ & $136(3)$ \\
\hline N1B-H1NB $\cdots$ O2B & $0.80(3)$ & $2.04(3)$ & $2.647(3)$ & $133(3)$ \\
\hline C1A-H1AA $\cdots$ O1Bi & 0.980 & 2.590 & $3.512(4)$ & 156.00 \\
\hline C7A-H7AA $\cdots$ O1A & 0.980 & 2.230 & $2.985(4)$ & 133.00 \\
\hline C7B-H7BA $\cdots$ O1B & 0.980 & 2.210 & $2.974(4)$ & 134.00 \\
\hline C13A-H13A $\cdots$ O1Dii & 0.950 & 2.550 & $3.329(3)$ & 140.00 \\
\hline C13B-H13B $\cdots$ O1Ciii & 0.950 & & $3.340(3)$ & 153.00 \\
\hline C13D-H13D $\cdots$ O1B & 0.950 & 2.490 & $3.291(3)$ & 141.00 \\
\hline C1C-H1CA $\cdots$ OlCiv & 0.980 & 2.490 & $3.427(4)$ & 159.00 \\
\hline C7C-H7CA $\cdots$ O1C & 0.980 & 2.20 & $2.970(4)$ & 135.00 \\
\hline C7C-H7CB $\cdots$ O1Dv & 0.980 & 2.560 & $3.408(4)$ & 145.00 \\
\hline C7D-H7DA $\cdots$ O1D & 0.980 & 2.230 & $2.992(4)$ & 134.00 \\
\hline
\end{tabular}

Symmetry codes: (i) $x+1, y+1, z$; (ii) $-x+2,-y,-z+1$; (iii) $-x,-y+1$, $-z+2$; (iv) $-x-1,-y+1,-z+1$; (v) $-x+1,-y,-z+1$.
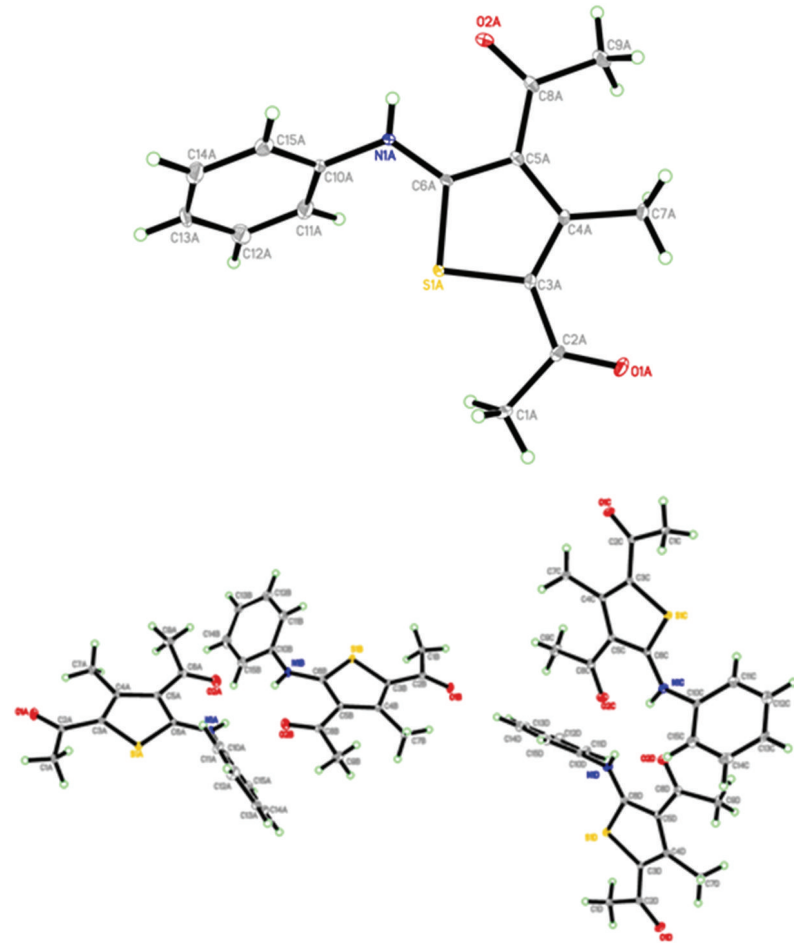

FIGURE 1: ORTEP of the synthesized compound 2.

Interestingly, enaminone 3 treated with $p$-chlorobenzoic acid to afford the corresponding ester 9. The IR spectrum is consistent with the expected absorption bands. ${ }^{1} \mathrm{H}-\mathrm{NMR}$ spectrum in DMSO- $d_{6}$ showed singlet at 3.12 related to the $\mathrm{CH}_{3}$ group and two doublets corresponding to the $\mathrm{CH}=\mathrm{CH}$ protons at $\delta 5.36(J=12.0 \mathrm{~Hz})$ and $5.36(J=12.0 \mathrm{~Hz})$ and the pyridinone $\mathrm{CH}$ protons at $\delta 6.15(J=8.0 \mathrm{~Hz})$ and 6.16

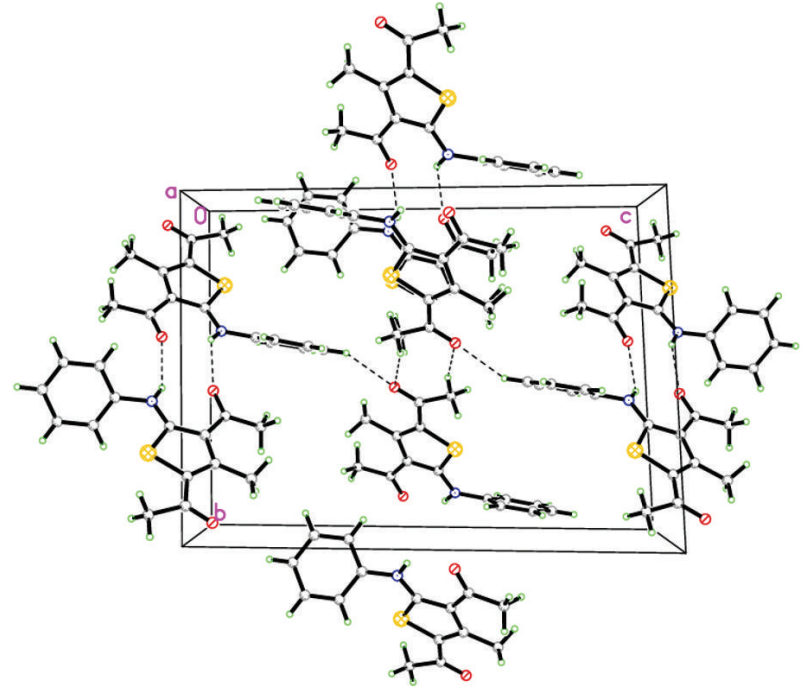

FIGURE 2: Molecular packing of the synthesized compound 2.

$(J=8.0 \mathrm{~Hz})$, in addition to a multiplet corresponding to the phenyl rings in the range $\delta 7.68-7.62 .{ }^{13} \mathrm{C}-\mathrm{NMR}$ spectrum is also in agreement with the suggested structure; it showed signals corresponding to the different carbon atoms in the compound as follows: $\delta 16.11\left(\mathrm{CH}_{3}\right), 115.13\left(\mathrm{CH}_{\mathrm{a}}\right), 126.33$ $\left(\mathrm{CH}_{, \mathrm{a}^{\prime}}\right), 128.58,130.54,130.95,132.60,137.24$ (Ar-C), 141.03 (SCCO), $142.34\left(-\mathrm{C}-\mathrm{CH}_{3}\right), 153.16\left(\mathrm{CH}_{\mathrm{b}^{\prime}}\right), 154.52(\mathrm{~S}-\mathrm{C}-\mathrm{N}), 176.51$ $(\mathrm{COO})$, and $180.51(\mathrm{C}=\mathrm{O})$. GC-MS exhibited the correct molecular ion peak $[\mathrm{M}]^{+}$at $m / z=449$ corresponding to the molecular formula $\mathrm{C}_{24} \mathrm{H}_{14} \mathrm{ClNO}_{4} \mathrm{~S}$.

\section{Conclusions}

In summary, we have succeeded in the synthesis of a number of novel thieno-fused bicyclic compounds via novel enaminone containing thieno[2,3-b]pyridine core by classical method. The structures of the new compounds were confirmed by means of different spectroscopic methods and by elemental analyses. The biological activity of the synthesized product is underway in our laboratory.

\section{Supplementary Material}

Supplementary Material will be available online at http:// dx.doi.org/10.1155/2015/382381.

\section{Conflict of Interests}

The authors have declared that there is no conflict of interests.

\section{Authors' Contribution}

The authors contributed equally to this work. 


\section{Acknowledgment}

The authors would like to extend their sincere appreciation to the Deanship of Scientific Research at king Saud University for funding this research group (no. RG -007-2015).

\section{References}

[1] S. Bondock, W. Fadaly, and M. A. Metwally, "Synthesis and antimicrobial activity of some new thiazole, thiophene and pyrazole derivatives containing benzothiazole moiety," European Journal of Medicinal Chemistry, vol. 45, no. 9, pp. 36923701, 2010.

[2] H. M. Hassneen and T. A. Abdallah, "New routes to pyridino $[2,3-d]$ pyrimidin-4-one and pyridino-[2,3-d]triazolino[4,5-a]pyrimidin-5-one derivatives," Molecules, vol. 8, no. 3, pp. 333-341, 2003.

[3] Y. N. Mabkhot, A. M. Al-Majid, and A. S. Alamary, "Synthesis and chemical characterisation of some new diheteroaryl thienothiophene derivatives," Molecules, vol.16, no. 9, pp. 77067714, 2011.

[4] K. M. Dawood, A. M. Farag, and Z. E. Kanddeel, "Heterocyclic synthesis via enaminonitriles: one-pot synthesis of some new pyrazole, isoxazole, pyrimidine, pyrazolo [1,5a]pyrimidine, pyrimido[1,2-a]benzimidazole and pyrido[1,2a] benzimidazole derivatives," Journal of Chemical Research, Synopses, no. 2, pp. 88-89, 1999.

[5] L. U. E. Ping and J. V. Greenhill, "Enaminones in heterocyclic synthesis," Advances in Heterocyclic Chemistry, no. 67, pp. 207343, 1997.

[6] P. G. Baraldi, A. Barco, S. Benetti, G. P. Pollini, and D. Simoni, "Synthesis of natural products via isoxazoles," Synthesis, no. 10, pp. 857-869, 1987.

[7] K. Saito, A. Nakao, T. Shinozuka et al., "Discovery and structure-activity relationship of thienopyridine derivatives as bone anabolic agents," Bioorganic and Medicinal Chemistry, vol. 21, no. 7, pp. 1628-1642, 2013.

[8] L. C. S. Pinheiro, J. C. Borges, C. D. Oliveira et al., "Synthesis of new 4-(phenylamino)thieno[2,3-b]pyridines and derivatives of the novel benzo $[b]$ thieno[3,2-h] $[1,6]$ naphthyridine tetracyclic system," Arkivoc, vol. 2008, no. 14, pp. 77-87, 2008.

[9] A. M. R. Bernardino, L. C. D. S. Pinheiro, C. R. Rodrigues et al., "Design, synthesis, SAR, and biological evaluation of new 4-(phenylamino)thieno[2,3-b]pyridine derivatives," Bioorganic and Medicinal Chemistry, vol. 14, no. 16, pp. 5765-5770, 2006.

[10] S. A. Al-Trawneh, M. M. El-Abadelah, J. A. Zahra et al., "Synthesis and biological evaluation of tetracyclic thienopyridones as antibacterial and antitumor agents," Bioorganic and Medicinal Chemistry, vol. 19, no. 8, pp. 2541-2548, 2011.

[11] J.-P. Wu, R. Fleck, J. Brickwood et al., "The discovery of thienopyridine analogues as potent $\mathrm{I} \kappa \mathrm{B}$ kinase $\beta$ inhibitors. Part II," Bioorganic \& Medicinal Chemistry Letters, vol. 19, no. 19, pp. 5547-5551, 2009.

[12] J. W. Lockman, M. D. Reeder, K. Suzuki et al., "Inhibition of eEF2-K by thieno[2,3-b]pyridine analogues," Bioorganic and Medicinal Chemistry Letters, vol. 20, no. 7, pp. 2283-2286, 2010.

[13] M. E. Schnute, D. J. Anderson, R. J. Brideau et al., "2-Aryl-2hydroxyethylamine substituted 4-oxo-4,7-dihydrothieno[2,3b] pyridines as broad-spectrum inhibitors of human herpesvirus polymerases," Bioorganic and Medicinal Chemistry Letters, vol. 17, no. 12, pp. 3349-3353, 2007.
[14] H. Liu, Y. Li, X.-Y. Wang et al., "Synthesis, preliminary structure-activity relationships, and in vitro biological evaluation of 6-aryl-3-amino-thieno[2,3-b]pyridine derivatives as potential anti-inflammatory agents," Bioorganic and Medicinal Chemistry Letters, vol. 23, no. 8, pp. 2349-2352, 2013.

[15] I. Adachi, T. Yamamori, Y. Hiramatsu et al., "Studies on dihydropyridines. III. Synthesis of 4,7-dihydrothieno[2,3-b]pyridines with vasodilator and antihypertensive activities," Chemical and Pharmaceutical Bulletin, vol. 36, no. 11, pp. 43894402, 1988.

[16] M. M. El-Abadelah, S. S. Sabri, H. A. Al-Ashqar, P. Mion, J. Bompart, and M. Calas, "Thienopyridinone antibacterials. Part II. ${ }^{1}$ Synthesis and antibacterial activity of some 2-chloro7-cyclopropyl-4,7-dihydro-4-oxothieno [2,3-b]pyridine-5-carboxylic acids," Phosphorus, Sulfur, and Silicon and the Related Elements, vol. 134, no. 1, pp. 21-29, 1998.

[17] E. R. Bacon and S. J. Daun, "Synthesis of 7-ethyl-4,7-dihydro4-oxo-2-(4-pyridinyl)thieno[2,3-b]pyridine-5-carboxylic acid," Journal of Heterocyclic Chemistry, vol. 28, no. 8, pp. 1953-1955, 1991.

[18] Y. N. Mabkhoot, "Synthesis and analysis of some bis-heterocyclic compounds containing sulphur," Molecules, vol. 14, no. 5, pp. 1904-1914, 2009.

[19] Y. N. Mabkhoot, "Synthesis and chemical characterisation of new bis-thieno $[2,3-b]$ thiophene derivatives," Molecules, vol. 15, no. 5, pp. 3329-3337, 2010.

[20] Y. N. Mabkhot, N. A. E. Kheder, and A. M. Al-Majid, "Facile and convenient synthesis of new thieno[2,3-b]-thiophene derivatives," Molecules, vol. 15, no. 12, pp. 9418-9426, 2010.

[21] Y. N. Mabkhot, F. D. Aldawsari, S. S. Al-Showiman et al., "Synthesis, bioactivity, molecular docking and POM analyses of novel substituted thieno[2,3-b]thiophenes and related congeners," Molecules, vol. 20, no. 2, pp. 1824-1841, 2015.

[22] Y. N. Mabkhot, N. N. E. El-sayed, F. Alatibi, A. Barakat, H. A. Ghabbour, and H.-K. Fun, "Synthesis, characterization and X-ray crystal structure of 3-methyl-4-oxo-7-phenyl-4,7dihydrothieno[2,3-b]pyridine-2-carboxyxlic acid ethyl ester," Asian Journal of Chemistry, vol. 26, no. 24, pp. 8596-8598, 2014.

[23] G. M. Sheldrick, "A short history of SHELX”, Acta Crystallographica Section A, vol. 64, no. 1, pp. 112-122, 2008.

[24] Brucker, APEX2, SAINT and SADABS, Bruker, Madison, Wis, USA, 2009. 

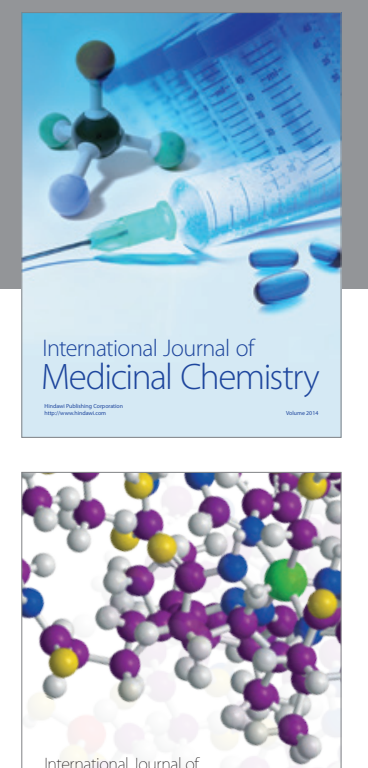

\section{Carbohydrate} Chemistry

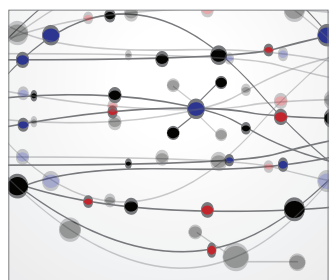

The Scientific World Journal
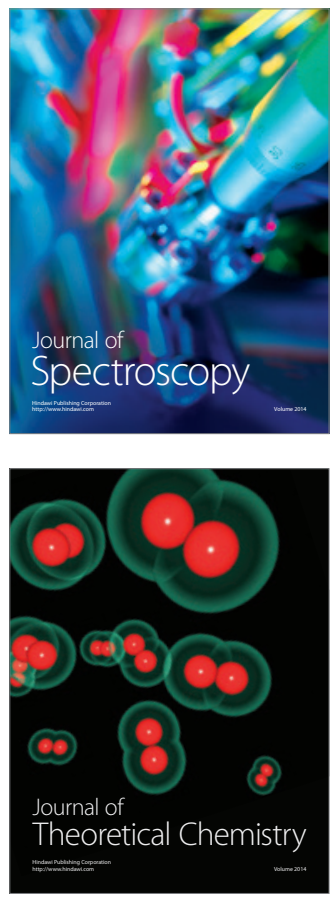
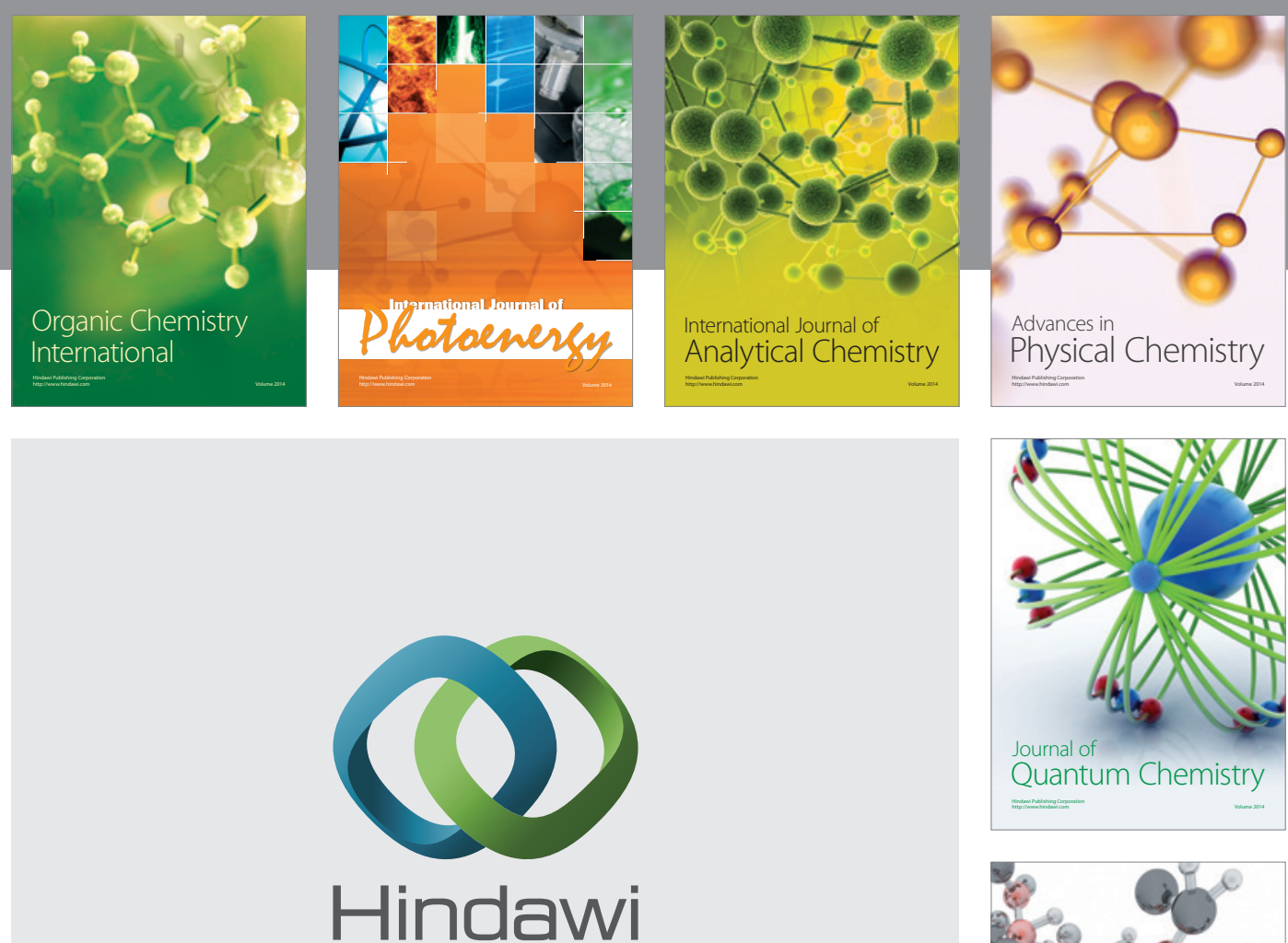

Submit your manuscripts at

http://www.hindawi.com

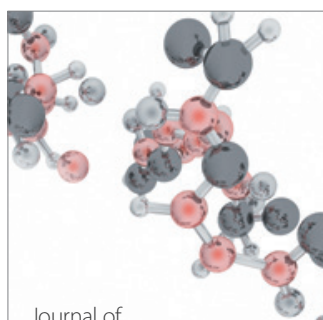

Analytical Methods

in Chemistry

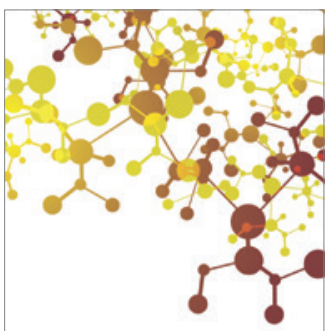

Journal of

Applied Chemistry

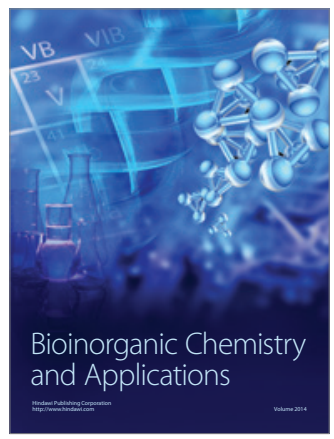

Inorganic Chemistry
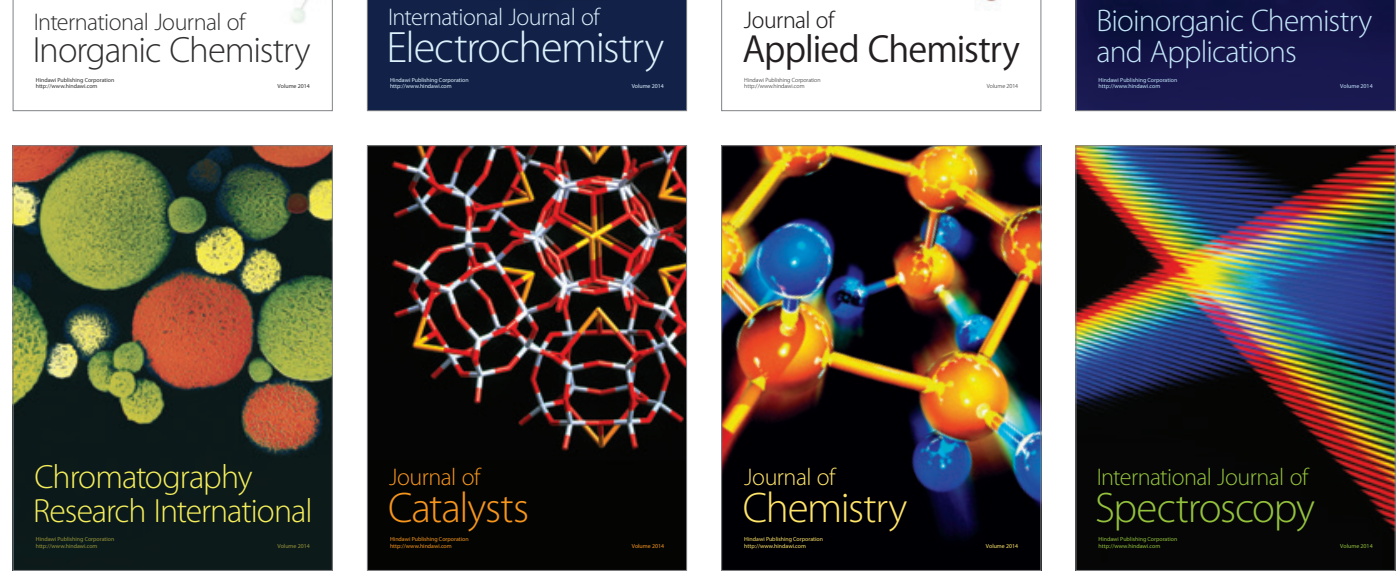